\title{
SEMICONVEX SPACES
}

\author{
by S. O. IYAHEN
}

(Received 24 June, 1967)

1. Introduction. Many of the techniques and notions used to study various important theorems in locally convex spaces are not effective for general linear topological spaces. In [4], a study is made of notions in general linear topological spaces which can be used to replace barrelled, bornological, and quasi-barrelled spaces. The present paper contains a parallel study in the context of semiconvex spaces.

In section 2, we look briefly and generally at semiconvex spaces, and answer a question of S. Simons [11, p. 180]. We then define the notion of an $s c$-inductive limit of semiconvex spaces -a concept which plays much the same part in the theory of semiconvex spaces as an inductive limit does for locally convex spaces. In section 3, we study hyperbarrelled, hyperbornological and quasi-hyperbarrelled spaces. These respectively replace barrelled, bornological and quasi-barrelled spaces when semiconvex spaces are being considered. The $\mathbf{N}$-hyperbarrelled and $N$-quasi-hyperbarrelled spaces introduced in section 4 are slight generalizations of what replace countably barrelled and countably quasi-barrelled spaces in a similar situation. Throughout, we shall use the terminology and notation of [4].

The author wishes to thank Professor A. P. Robertson for his help and encouragement. $\mathrm{He}$ is grateful to the Government of the Republic of Nigeria for financial support.

2. Semiconvex spaces. Let $E$ be a linear space. If $\lambda$ is a non-negative real number, a subset $A$ of $E$ is called $\lambda$-convex if $A+A \subseteq \lambda A$. A subset $B$ of $E$ is called a semiconvex subset if it is $\mu$-convex for some $\mu \geqq 0$. A linear topology $u$, say, on $E$ with a base of balanced semiconvex neighbourhoods of the origin is called a semiconvex topology and $(E, u)$ is known as a semiconvex linear topological space (1.t.s.) or more shortly as a semiconvex space.

Any locally convex or locally bounded space is a semiconvex space. A subspace of a semiconvex space is a semiconvex space and so is any product of semiconvex spaces. It is easy to see from the proof of Theorem 1 of [9], that a separated 1.t.s. is a semiconvex space if and only if it is topologically isomorphic to a subspace of a product of (complete) separated locally bounded spaces.

Since the upper bound of any set of semiconvex topologies on a linear space is semiconvex, there exists a finest semiconvex topology on any linear space $E$. This will be denoted by $s c$. A base of neighbourhoods for $s c$ is the family of all balanced semiconvex absorbent subsets of $E$. Clearly $\tau\left(E, E^{*}\right)$ is coarser than $s c$ and $s c$ is coarser than the finest linear topology $s$ on $E$, the three topologies coinciding when the dimension of $E$ is countable. When the dimension of $E$ is uncountable $\tau\left(E, E^{*}\right), s c$ and $s$ are distinct. For let $G$ be the sequence space $l^{\frac{1}{2}}$ and $H$ the space of all Lebesgue measurable functions on the closed interval $[0,1]$, with the metrizable topology corresponding to convergence in measure. Since $G$ and $H$ have the same dimension $\left(2{ }^{*}\right)$, they are algebraically isomorphic and we may identify them. That $\tau\left(G, G^{*}\right)$ is strictly coarser than $s c$ follows from the fact that the topology of $l^{\frac{1}{2}}$ cannot be coarser than $\tau\left(G, G^{*}\right)$. 
As $H$ is not a semiconvex space [8, p. 239], and any continuous nearly open linear image of a semiconvex space is obviously a semiconvex space, we deduce that the topology of $H$ is not coarser than $s c$. Thus $s c$ is strictly coarser than $s$. If $E$ is any linear space of uncountable dimension, $G$ may be identified with a linear subspace of $E$, and from this the assertion follows.

According to S. Simons [11, p. 170], a function $f$ on a linear space $E$ into the non-negative real numbers is called an $r$-pseudometric $(0<r \leqq 1)$ if

(i) there exists $x$ in $E$ such that $f(x) \neq 0$,

(ii) $f(x+y) \leqq f(x)+f(y)$ for all $x, y$ in $E$, and

(iii) $f(\lambda x)=|\lambda| r f(x)$ for each $x$ in $E$ and $\lambda$ in the scalar field.

By an application of Theorem 1 of [11] we see that if, for each $r$ in $0<r \leqq 1,\left(f_{\alpha}: \alpha \in \Phi_{r}\right)$ is the set of all $r$-pseudometrics on a linear space $E$ and $\Phi=\bigcup_{0<r \leqq 1} \Phi_{r}$, then a base of neighbourhoods for $s c$ is the family of sets $\left(f_{\alpha}^{-1}\left[0, n^{-1}\right]: \alpha \in \Phi, n=1,2, \ldots\right)$. Now, by using $\left(f_{\alpha}: \alpha \in \Phi\right)$ in place of invariant pseudometrics $q$ in problem $E$, page 124 of [7], one can easily show that for any linear space $E,(E, s c)$ is complete.

The notion of S. Simons for an upper bound space in [11] clearly coincides with that of a semiconvex space. If $E$ is an uncountably dimensional linear space, then, under its finest linear topology $s, E$ is not an upper bound space, since $s$ is strictly finer than $s c$. Let $B$ be a subset of $E$ such that for every s-continuous pseudometric $f$ on $E, f(B)$ is bounded. (Any point of $E$ is such a $B$.) This trivially implies that for every $s c$-continuous pseudometric $f$ on $E, f(B)$ is bounded. Thus by Theorem 6 of [11], $B$ is $s c$-bounded. Since $s, s c$ have the same bounded sets, $B$ must be $s$-bounded. This answers (in the negative) a question of S. Simons [11, p. 180].

Let $E$ be a linear space, and suppose that for each $\gamma$ in an index set $\Gamma, E_{\gamma}$ is a semiconvex space and $u_{\gamma}$ is a linear map of $E_{\gamma}$ into $E$ such that the union of the subspaces $u_{\gamma}\left(E_{\gamma}\right)$ spans $E$. The upper bound $\mathscr{T}$, say of all semiconvex topologies on $E$ for which each $u_{y}$ is continuous, is the finest semiconvex topology on $E$ for which each $u_{\gamma}$ is continuous. We shall call $\mathscr{T}$ the sc-inductive limit topology on $E$ induced by $\left(E_{\gamma} ; u_{\gamma}: \gamma \in \Gamma\right)$ and say that $(E, \mathscr{T})$ is the $s c-$ inductive limit of $\left(E_{\gamma} ; u_{\gamma}: \gamma \in \Gamma\right)$.

With the notation above, a base of neighbourhoods of the origin for the topology $\mathscr{T}$ is the family $\mathscr{U}$ of all balanced semiconvex subsets of $E$ such that for every $U$ in $\mathscr{U}, u_{\gamma}^{-1}(U)$ is a neighbourhood of the origin in $E_{\gamma}$ for each $\gamma$ in $\Gamma$. Also, a linear map $t$ from $(E, \mathscr{T})$ into a semiconvex space is continuous if and only if each $t_{0} u_{\gamma}$ is continuous.

If for a fixed $\lambda>0$, each $E_{\gamma}$ has a base of balanced $\lambda$-convex neighbourhoods of the origin and $\Gamma$ is countable, then, by Proposition 2.2 of [4], the space $(E, \mathscr{T})$ referred to above is the *-inductive limit of $\left(E_{\gamma} ; u_{\gamma}: \gamma \in \Gamma\right)$. However, since, for an uncountably dimensional linear space $E$ the topologies $\tau\left(E, E^{*}\right), s c$ and $s$ are distinct and are respectively the inductive limit topology, sc-inductive limit topology and $*$-inductive limit topology of some $\left(K_{\gamma} ; u_{\gamma}: \gamma \in \Gamma\right)$ (each $K_{y}$ is a copy of the scalar field), we see that an $s c$-inductive limit of locally convex spaces need not be locally convex and that a $*$-inductive limit of locally convex spaces need not be a semiconvex space. 
3. Hyperbarrelled, hyperbornological and quasi-hyperbarrelled spaces. Let $E$ be a linear space and $u, v, w$ semiconvex topologies on $E$. If every $u$-closed balanced semiconvex absorbent subset of $E$ is a $u$-neighbourhood of the origin, we say that $(E, u)$ is hyperbarrelled. We call $(E, v)$ hyperbornological if every bounded linear map from $(E, v)$ into any semiconvex space is continuous. We say that $(E, w)$ is quasi-hyperbarrelled if every $w$-closed balanced semiconvex $w$-bornivorous subset is a $w$-neighbourhood of the origin.

Clearly if $(E, u)$ is ultrabarrelled (ultrabornological, quasi-ultrabarrelled) and $u^{0}$ is the finest semiconvex topology on $E$ coarser than $u$, then $\left(E, u^{0}\right)$ is hyperbarrelled (hyperbornological, quasi-hyperbarrelled). Also, if $(F, v)$ is hyperbarrelled (hyperbornological, quasihyperbarrelled), then $\left(F, v^{00}\right)$ is barrelled (bornological, quasi-barrelled). In particular, every semiconvex ultrabarrelled (ultrabornological, quasi-ultrabarrelled) space is hyperbarrelled (hyperbornological, quasi-hyperbarrelled), and every locally convex hyperbarrelled (hyperbornological, quasi-hyperbarrelled) space is barrelled (bornological, quasi-barrelled).

Let $T$ be a set of linear maps from an l.t.s. $E$ to an 1.t.s. $F$, and let $B$ be a balanced semiconvex bornivorous subset of $F$. The set $\bigcap_{t \in T} t^{-1}(B)$ is balanced and semiconvex. It is absorbent in $E$ if $T$ is pointwise bounded, and bornivorous if $T$ is uniformly bounded on bounded sets. If $B$ is closed and each member of $T$ is continuous, $\bigcap_{t \in T} t^{-1}(B)$ is also closed. The following result can be easily deduced from these observations.

THEOREM 3.1. Let $T$ be a set of linear maps from one semiconvex space $E$ to another. Then $T$ is equicontinuous if

(a) $E$ is hyperbarrelled and $T$ is a pointwise bounded set of continuous linear maps, or if

(b) $E$ is hyperbornological and $T$ is uniformly bounded on bounded sets, or if

(c) $E$ is quasi-hyperbarrelled and $T$ is a set of continuous linear maps which is uniformly bounded on bounded sets.

The proof of the following result is straightforward.

THEOREM 3.2. Any sc-inductive limit of hyperbarrelled (hyperbornological, quasi-hyperbarrelled) spaces is of the same sort. In particular, any sc-inductive limit of semiconvex complete metric linear spaces has all three properties.

COROLLARY 1. Every quotient by a linear subspace of a hyperbarrelled (hyperbornological, quasi-hyperbarrelled) space is of the same sort.

COROLLARY 2. Every product of finitely many hyperbarrelled (hyperbornological, quasihyperbarrelled) spaces is of the same sort.

COROLlaRy 3. Every countable inductive limit of locally convex hyperbarrelled (hyperbornological, quasi-hyperbarrelled) spaces has the same property. In particular, every countable inductive limit of Fréchet spaces has all three properties.

Since a countably infinite dimensional normed linear space is not barrelled, it follows that a hyperbornological space need not be hyperbarrelled. Let $E$ be an incomplete separated inductive limit of a sequence of Banach spaces and let $x$ be a point belonging to the completion $E^{\wedge}$ of $E$ but not in $E$. As was pointed out in [4], the linear subspace $E_{1}$ of $E$ spanned by $E$ and 
$x$ is ultrabarrelled, and thus $E_{1}$ is hyperbarrelled. But it is not hyperbornological, since, by [10, p. 155], it is not bornological. Let $F$ be a hyperbarrelled space which is not hyperbornological and $G$ a hyperbornological space which is not hyperbarrelled. Then, by Corollary 2 of Theorem 3.2, $F \times G$ is a quasi-hyperbarrelled space, which, in view of Corollary 1 of the same theorem, is neither hyperbarrelled nor hyperbornological.

It is easy to show that a linear map from a hyperbarrelled space into a semiconvex space is nearly continuous. Also, a bounded linear map from a quasi-hyperbarrelled space into a semiconvex space is nearly continuous. Since, by Corollary 1 of Theorem 3.2, every quotient by a linear subspace of a hyperbarrelled (quasi-hyperbarrelled) space is of the same sort, we deduce from a result in page 213 of [6] and the argument preceding Theorem 3.2 of [4] that every closed (closed bounded) linear map from a hyperbarrelled (quasi-hyperbarrelled) space into a semiconvex complete metric linear space is continuous. In particular, every closed (closed bounded) linear map from a hyperbarrelled (quasi-hyperbarrelled) space into a complete separated locally bounded space is continuous.

Let $B$ be a closed balanced semiconvex absorbent subset of a semiconvex space $(E, v)$. Then $B$ is $\lambda$-convex for some $\lambda>0$. Let $N$ be the intersection of $\left(\lambda^{-n} B: n=1,2, \ldots\right)$ and let $k$ be the quotient map of $E$ onto $E / N$. The family of sets $\left(\lambda^{-n} k(B): n=1,2, \ldots\right)$ is a local base for a separated locally bounded linear topology $w$, say, on $E / N$. By Lemma 3.1 of [4], the graph of $k$ is closed in $(E, v) \times(E / N, w)^{\wedge}$ and $k$ is bounded if $B$ is $v$-bornivorous. Therefore if every closed linear map from $(E, v)$ into any complete separated locally bounded space is continuous, $B$ must be a $v$-neighbourhood of the origin; this implies that $(E, v)$ is hyperbarrelled. If alternatively, it is given that every closed bounded linear map from $(E, v)$ into any complete separated locally bounded space is continuous, then $(E, v)$ is quasi-hyperbarrelled. Just as in Theorem 4.2 of [4], if every bounded linear map from a semiconvex space $E$ into any complete separated locally bounded space is continuous, $E$ is hyperbornological. We note these for further reference.

THEOREM 3.3. A semiconvex space $E$ is hyperbarrelled (hyperbornological, quasi-hyperbarrelled) if and only if every closed (bounded, closed bounded) linear map from $E$ into any complete separated locally bounded space is continuous.

LEMMA 3.1. Iff is a closed linear map from a product $\underset{\gamma \in \Phi}{\times} E_{y}$ of separated linear topological spaces into a complete separated locally bounded space $F$, then there is a finite subset $\Phi_{0}$ of $\Phi$ such that the restriction of $f$ to $X\left(E_{\gamma}: \gamma \in \Phi \mid \Phi_{0}\right)$ is the zero map.

Proof. It is sufficient to show that for some finite subset $\Phi_{0}$ of $\Phi$, if $\gamma \in \Phi_{1}=\Phi \mid \Phi_{0}$, then the restriction of $f$ to $E_{\gamma}$ is zero. For, if this is so, $\sum_{\gamma \in \Phi_{1}} E_{\gamma} \subseteq f_{1}^{-1}(0)$, where $f_{1}$ is the restriction of $f$ to $\underset{\gamma \in \Phi_{1}}{X} E_{\gamma}$. Now $f_{1}^{-1}(0)$ is closed in $\underset{\gamma \in \Phi_{1}}{X} E_{\gamma}$, since the graph of $f_{1}$ is closed in $\underset{\gamma \in \Phi_{1}}{X} E_{\gamma} \times F$. And as $\sum_{\gamma \in \Phi_{1}} E_{\gamma}$ is dense in $\underset{\gamma \in \Phi_{1}}{X} E_{\gamma}$, it follows that $f_{1}^{-1}(0)=\underset{\gamma \in \Phi_{1}}{X} E_{\gamma}$.

Let $|\ldots|$ denote a quasinorm on $F$ which defines its topology. If there is no finite subset $\Phi_{0}$ of $\Phi$ such that for every $\gamma$ in $\Phi \mid \Phi_{0}, f$ is the zero map on $E_{\gamma}$, then, for some sequence $\left(\gamma_{i}: i=1,2, \ldots\right)$ of distinct members of $\Phi$, there exist points $\left(x_{\gamma_{i}}\right)$ such that $x_{\gamma_{i}}$ is in $E_{\gamma_{i}}$ and $\left|f\left(x_{\gamma_{1}}\right)\right|=i$. Clearly, $\left(f\left(x_{y_{i}}\right)\right)$ is not bounded in $F$. 
Now, $\underset{\gamma \in \Phi}{X} E_{y}$ induces the product topology on $G=\mathrm{X}\left(K_{\gamma_{i}} x_{\gamma_{i}}\right)\left(K_{y_{i}}\right.$ is a copy of the scalar field for each $i$ ). Moreover the restriction of $f$ to the Fréchet space $G$ has a graph closed in $G \times F$, and is therefore continuous by Banach's closed graph theorem. This implies that $\left(f\left(x_{\gamma_{i}}\right)\right)$ is bounded in $F$. From this contradiction, the result follows.

Since, by Corollary 2 of Theorem 3.2, any finite product of hyperbarrelled (quasi-hyperbarrelled) spaces is of the same sort, the following result is immediate, on using Lemma 3.1 and Theorem 3.3.

THEOREM 3.4. Any separated product of hyperbarrelled (quasi-hyperbarrelled) spaces is hyperbarrelled (quasi-hyperbarrelled).

If $E_{0}$ is a subspace of an l.t.s. $E$ of finite co-dimension, then, as shown in Proposition 3.1 of [4], any closed linear map from $E_{0}$ into an l.t.s. has a closed linear extension to all of $E$. It therefore follows from Theorem 3.3 that any subspace of finite co-dimension of a hyperbarrelled space is hyperbarrelled. In particular, any hyperplane in a hyperbarrelled space is of the same sort. Also, any separated semiconvex space is topologically isomorphic to a subspace of a product of complete separated locally bounded spaces. Thus any separated semiconvex space is a subspace of some separated hyperbarrelled space. The proof of the following result uses these observations and a method due to Komura [10, Theorem 1.1].

THEOREM 3.5. Every separated semiconvex space is a closed subspace of some separated hyperbarrelled space.

Proof. Let $E$ be a separated semiconvex space. If $F$ is a separated hyperbarrelled space containing $E$, let $\left(e_{\alpha}: \alpha \in \Psi\right)$ be a Hamel basis for an algebraic supplement of $E$ in $F$. For each $\beta$ in $\Psi$, let $F_{\beta}$ be the linear subspace of $F$ spanned by $E$ and $\left(e_{\alpha}: \alpha \neq \beta, \alpha \in \Psi\right)$. Clearly $E=\bigcap_{\beta \in \Psi} F_{\beta}$, and each $F_{\beta}$ is hyperbarrelled. It is easy to show that the map $f$ of $E$ into $G=\underset{\beta \in \Psi}{X} F_{\beta}$, defined as follows, is a topological isomorphism: for each $x$ in $E, f(x)=\left(x_{\beta}\right)$, where $x_{\beta}=x$ for all $\beta$ in $\Psi$. So all that remains is to show that $f(E)$ is closed in $G$. If $\left(\left(x_{\alpha}\right)_{\gamma}: \gamma \in \Phi\right)$ is a net in $f(E)$ converging to $\left(y_{\alpha}\right)$ in $G$, then, for each $\alpha\left(=\alpha_{0}\right.$, say) in $\Psi$, $\left(x_{\alpha_{0} \gamma}: \gamma \in \Phi\right)$ is a net in $E$ converging to $y_{\alpha_{0}}$ in $F_{\alpha_{0}}$. Since by the definition of $f, x_{\alpha_{1} \gamma}=x_{\alpha_{2} \gamma}$ for any $\alpha_{1}, \alpha_{2}$ in $\Psi$, it follows that for each $\alpha$ in $\Psi, y_{\alpha} \in \bigcap_{\alpha \in \Psi} F_{\alpha}(=E)$ and that $\left(y_{\alpha}\right) \in f(E)$. Hence $f(E)$ is closed in $G$, which, by Theorem 3.4, is hyperbarrelled.

Let $\left(E_{\gamma}: \gamma \in \Phi\right)$ be a family of separated bornological spaces. If $K^{\Phi}(K$ is the scalar field) is bornological, so is $\underset{\gamma \in \Phi}{X} E_{\gamma}$. Bourbaki's proof of this assertion in [1, p. 15, exercise 18b] uses the fact that a separated locally convex space $E$ is bornological if every bounded linear map from $E$ into any Banach space is continuous. By using Theorem 3.3 in place of this in Bourbaki's argument, we see that the result stated above holds with "hyperbornological" replacing " bornological". In particular, a countable product of separated hyperbornological spaces is hyperbornological.

As defined in [4], an 1.t.s. is said to be almost convex if every bounded subset is contained in some bounded set which is closed, balanced and semiconvex. Since every bounded set in any linear space under its finest linear topology is contained in a closed absolutely convex 
bounded set, we deduce that an almost convex 1.t.s. need not be a semiconvex space. The following result is an analogue of Theorem 5.2 of [4]. The proof is omitted, being similar to that of the corresponding result in [4].

THEOREM 3.6. A separated sequentially complete almost convex quasi-hyperbarrelled space is hyperbarrelled.

Let $E$ be a separated almost convex hyperbornological space. By an argument similar to that after Theorem 5.2 of [4], one can show that the space $E$ is an $s c$-inductive limit of separated locally bounded spaces. If $E$ is sequentially complete, then it is an $s c$-inductive limit of complete separated locally bounded spaces and is therefore hyperbarrelled.

4. $\aleph$-hyperbarrelled and $\aleph$-quasi-hyperbarrelled spaces. We say that a semiconvex space is $\mathbf{\aleph - h y p e r b a r r e l l e d ~ ( ~} ゙$-quasi-hyperbarrelled) if every closed balanced semiconvex absorbent (bornivorous) subset $V$ is a neighbourhood of the origin whenever it satisfies the following condition:

$V=\bigcap_{\gamma \in \Phi} U_{\gamma}$, where, for some $\lambda>0$, each $U_{\gamma}$ is a closed balanced $\lambda$-convex neighbourhood and the cardinal of $\Phi$ is $\aleph$.

Clearly any hyperbarrelled (quasi-hyperbarrelled, $\aleph$-hyperbarrelled) space is $\boldsymbol{N}$-hyperbarrelled (ぶ-quasi-hyperbarrelled, $\aleph$-quasi-hyperbarrelled) for every $\aleph$. If $\aleph_{\alpha} \leqq \aleph_{\beta}$, then every $\aleph_{\beta}$-hyperbarrelled $\left(\aleph_{\beta}\right.$-quasi-hyperbarrelled) space is $\aleph_{\alpha}$-hyperbarrelled $\left(\aleph_{\alpha}\right.$-quasi-hyperbarrelled). By using Theorem 1 (Theorem 2) of [3] we see that every locally convex $\aleph_{0^{-}}$ hyperbarrelled $\left(\aleph_{0}\right.$-quasi-hyperbarrelled) space is countably barrelled (countably quasibarrelled). It therefore follows from example (ii) of [5] that for each $\aleph \geqq \aleph_{0}$, an $\aleph$-quasihyperbarrelled space need not be $\mathbf{N}$-hyperbarrelled. Also, by using example (iii) of [5] and Theorem 3.5, we see that a closed linear subspace of an $\aleph$-hyperbarrelled ( $\aleph$-quasi-hyperbarrelled) space need not be of the same sort.

If $\left(f_{\gamma}: \gamma \in \Phi\right)$ is a family of continuous linear maps from an 1.t.s. $(E, u)$ into an 1.t.s. $F$, then, for every closed balanced semiconvex ( $\lambda$-convex, say) neighbourhood $V$ of the origin in $F$, each $f_{\gamma}^{-1}(V)$ is a closed balanced $\lambda$-convex $u$-neighbourhood. If $\left(f_{\gamma}: \gamma \in \Phi\right)$ is pointwise bounded, then $\bigcap_{\gamma \in \Phi} f_{\gamma}^{-1}(V)$ is absorbent; it is bornivorous if $\left(f_{\gamma}: \gamma \in \Phi\right)$ is uniformly bounded on bounded sets. Using these observations, one can prove the following result.

THEOREM 4.1. Let $\Phi$ be an index set of cardinal $\aleph$, and let $\left(f_{\gamma}: \gamma \in \Phi\right)$ be a set of continuous linear maps from one semiconvex space $E$ to another. Then $\left(f_{\gamma}: \gamma \in \Phi\right)$ is equicontinuous if

(a) $E$ is $\aleph$-hyperbarrelled and $\left(f_{\gamma}: \gamma \in \Phi\right)$ is pointwise bounded, or if

(b) $E$ is $\aleph$-quasi-hyperbarrelled and $\left(f_{\gamma}: \gamma \in \Phi\right)$ is uniformly bounded on bounded sets.

The following corollary is a direct consequence of the above theorem and J. D. Weston's main result in [13].

COROLLARY. If $\left(f_{n}\right)$ is a pointwise convergent sequence of continuous linear maps from an $\aleph_{0}$-hyperbarrelled space $E$ into a semiconvex space $F$, then its limit mapping is continuous. If in addition $F$ is sequentially complete, then $\left(f_{n}\right)$ is necessarily pointwise convergent if it is pointwise bounded on a set which is everywhere dense in $E$.

Let $(E, u)$ be the sequence space $l^{\frac{1}{2}}$. For each $x=\left(x_{1}, x_{2}, \ldots\right)$ in $E$, let $t_{n}(x)$ be $\left(x_{1}, x_{2}, \ldots\right.$ 
$\left.x_{n}, 0,0, \ldots\right)$. Then, as was pointed out in page 256 of [12], $\left(t_{n}\right)$ is a sequence of continuous linear maps from $\left(E, u^{00}\right)$ into $(E, u)$ such that, for each $x$ in $E, t_{n}(x)$ converges to $x$ in $(E, u)$. As the identity map from $\left(E, u^{\infty}\right)$ into $(E, u)$ is not continuous, it follows from the above corollary that the barrelled normed space $\left(E, u^{\infty}\right)$ is not $\aleph_{0}$-hyperbarrelled. Also, as was shown just before Theorem 5.1 of $[4],\left(t_{n}\right)$ is a sequence of continuous linear maps from $\left(E, \tau\left(E, E^{*}\right)\right)$ into $(E, u)$, which is uniformly bounded on bounded sets, but is not equicontinuous. Thus, by Theorem 4.1, $\left(E, \tau\left(E, E^{*}\right)\right)$ is not $\kappa_{0}$-quasi-hyperbarrelled. This implies that a separated inductive limit of Banach spaces need not be $\aleph_{0}$-quasi-hyperbarrelled. However, we have the following result.

THEOREM 4.2. Any sc-inductive limit of $\aleph$-hyperbarrelled ( $\aleph$-quasi-hyperbarrelled) spaces is of the same sort.

Proof. Let $(F, v)$ be the $s c$-inductive limit of $\left(E_{\alpha} ; u_{\alpha}: \alpha \in \Psi\right)$, where each $E_{\alpha}$ is $\aleph$-hyperbarrelled.

Let $V=\bigcap_{\gamma \in \Phi} V_{y}$, where, for some $\lambda>0$, each $V_{y}$ is a closed balanced $\lambda$-convex $v$-neighbourhood and the cardinal of $\Phi$ is $\aleph$. Suppose also that $V$ is absorbent; then, for each $\gamma$ in $\Phi$, each $u_{\alpha}^{-1}(V)$ is a closed balanced $\lambda$-convex neighbourhood of the origin in $E_{\alpha}$. Since $E_{\alpha}$ is $\aleph$-hyperbarrelled, $u_{\alpha}^{-1}(V)$ is a neighbourhood in $E_{\alpha}$. As this is true for all $\alpha$ in $\Psi, V$ is a $v$ neighbourhood. Thus $(F, v)$ is $\aleph$-hyperbarrelled.

Similarly, any $s c$-inductive limit of $\aleph$-quasi-hyperbarrelled spaces is of the same sort.

COROLLARY. Any countable inductive limit of locally convex $\aleph$-hyperbarrelled (ふ-quasihyperbarrelled) spaces is of the same sort.

LEMMA 4.1. If $B$ is a closed balanced semiconvex absorbent subset of a product $E=\underset{\gamma \in \Phi}{X} E_{\gamma}$ of semiconvex spaces, then there is a finite subset $\Phi_{0}$ of $\Phi$ such that $X\left(E_{\gamma}: \gamma \in \Phi \mid \Phi_{0}\right) \subseteq B$.

Proof. Let $F_{\gamma}$ denote $E_{\gamma}$ under its finest semiconvex topology. By Theorem 3.4, the space $(F, v)=\underset{\gamma \in \Phi}{X} F_{\gamma}$ is hyperbarrelled. Since $v$ is finer than the topology of $E, B$ is $v$-closed and must therefore be a $v$-neighbourhood of the origin. This gives the result.

In the above lemma, $E$ is the sc-inductive limit of $\underset{\gamma \in \Phi_{0}}{X} E_{\gamma}$ and $X\left(E_{\gamma}: \gamma \in \Phi \mid \Phi_{0}\right)$. Therefore

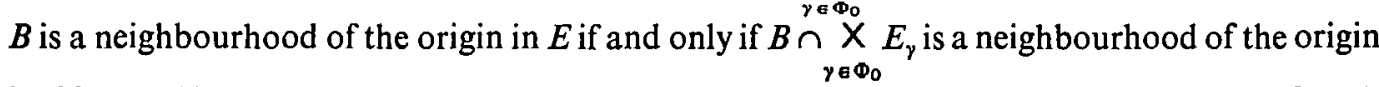
in $\underset{\gamma \in \Phi_{0}}{X} E_{\gamma}$. Since, by Theorem 4.2, $\underset{\gamma \in \Phi_{0}}{X} E_{\gamma}$ is $\aleph$-hyperbarrelled (ぶ-quasi-hyperbarrelled) if each $E_{\gamma}$ is, Theorem 4.3 follows easily.

THEOREM 4.3. Any product of $\aleph$-hyperbarrelled ( $\aleph$-quasi-hyperbarrelled) spaces has the same property.

By an argument similar to the one above, one can prove that a product of countably barrelled (countably quasi-barrelled) spaces is of the same sort.

The method used in the following lemma is a slight modification of that in [7, 22.9].

LEMMA 4.2. In the strong dual of a metrizable locally convex space, any bornivorous set which is the intersection of a sequence of balanced $\lambda$-convex neighbourhoods for some $\lambda>0$, is itself a neighbourhood of the origin. 
Proof. Let $E$ be a metrizable locally convex space with dual $E^{\prime}$. We shall suppose that $E^{\prime}$ has the strong topology $\beta\left(E^{\prime}, E\right)$. Let $U=\bigcap_{n \geq 1} U_{n}$, where each $U_{n}$ is a balanced $\lambda$-convex neighbourhood in $E^{\prime}$, be a bornivorous set in $E^{\prime}$. To prove that $U$ is a neighbourhood of the origin in $E^{\prime}$, it is sufficient to show that there exists a $\sigma\left(E^{\prime}, E\right)$-closed absolutely convex subset of $U$ which is absorbent in $E^{\prime}$. By 22.3 of [7], there exists in $E^{\prime}$ a fundamental sequence $\left(B_{n}\right)$ of bounded sets such that each $B_{n}$ is absolutely convex and $\sigma\left(E^{\prime}, E\right)$-compact.

For each $n$, there is a positive number $t_{n}$ such that $t_{n} B_{n} \subseteq \lambda^{-n-1} U$. Moreover, the convex envelope $G_{n}$ of $\bigcup_{1 \leqq i \leqq n} t_{i} B_{i}$ is a $\sigma\left(E^{\prime}, E\right)$-compact absolutely convex subset of $\lambda^{-1} U$. Also, for each $n$, there is a $\sigma\left(E^{\prime}, E\right)$-closed absolutely convex neighbourhood $W_{n}$ which is contained in $\lambda^{-1} U_{n}$. If $V_{n}=G_{n}+W_{n}$, then $V_{n}$ is a $\sigma\left(E^{\prime}, E\right)$-closed absolutely convex neighbourhood in $E^{\prime}$ and $V_{n} \subseteq U_{n}$. Let $V$ be the intersection of $\left(V_{n}\right)$. Then $V$ is a $\sigma\left(E^{\prime}, E\right)$-closed absolutely convex subset of $U$. And $V$ is absorbent in $E^{\prime}$ since it absorbs each $B_{n}$.

By using an argument similar to that in Theorem 5.2 of [4], one can show that any sequentially complete almost convex $\aleph$-quasi-hyperbarrelled space is $\aleph$-hyperbarrelled. It therefore follows from Lemma 4.2 that the strong dual of any metrizable locally convex space is $\aleph_{0}$ hyperbarrelled, since such a space is necessarily complete. But by [2, pp. 71 and 88], the strong dual of a metrizable locally convex space need not be quasi-barrelled. It therefore follows that an $\aleph_{0}$-hyperbarrelled space need not be quasi-hyperbarrelled.

\section{REFERENCES}

1. N. Bourbaki, Élements de mathématique, Livre V; Espaces vectorials topologiques, Ch. III-V, Actualités Sci. Ind. 1229 (Paris, 1955).

2. A. Grothendieck, Sur les espaces (F) et (DF), Summa Brasil. Math. 3 (1954), 57-123.

3. T. Husain, Two new classes of locally convex spaces, Math. Ann. 166 (1966), 289-299.

4. S. O. Iyahen, On certain classes of linear topological spaces, Proc. London Math. Soc. (3) 18 (1968), 285-307.

5. S. O. Iyahen, Some remarks on countably barrelled and countably quasi-barrelled spaces, Proc. Edinburgh Math. Soc. (2) 15 (1967), 295-296.

6. J. L. Kelley, General topology (New York, 1955).

7. J. L. Kelley and I. Namioka, Linear topological spaces (New York, 1963).

8. V. L. Klee, Exotic topologies for linear spaces, Proc. Sympos. general topology and its relation to modern analysis and algebra (Prague, 1961), 238-249. 281-285.

9. V. L. Klee, Shrinkable neighbourhoods in Hausdorff linear spaces, Math. Ann. 14 (1960),

10. Y. Komura, On linear topological spaces, Kumamoto J. Sci. Ser. A 5 (1962), 148-157.

11. S. Simons, Boundedness in linear topological spaces, Trans. Amer. Math. Soc. 113 (1964), 169-180.

12. W. Robertson, Completions of topological vector spaces, Proc. London Math. Soc. (3) 8 (1958), 242-257.

13. J. D. Weston, The principle of equicontinuity for topological vector spaces, Proc. Univ. Durham Philos. Soc. Ser. $A 13$ (1957), 1-5.

The University,

KeELE 